\title{
Experimental method to characterize the strain dependent permeability of tissue engineering scaffolds
}

Naser Nasrollahzadeh and Dominique P. Pioletti*

\author{
Laboratory of Biomechanical Orthopedics \\ Institute of Bioengineering \\ École Polytechnique Fédérale de Lausanne (EPFL)-Switzerland
}

Submitted to the Journal of Biomechanics

Short Communication

\section{Revision 1}

* Corresponding author: Dominique Pioletti, PhD

EPFL/STI/IBI/LBO

Station 19

1015 Lausanne

Switzerland

Tel: +41216938341

Fax: +41216938660

Email:dominique.pioletti@epfl.ch 
2 Permeability is an overarching mechanical parameter encompassing the effects of porosity,

3 pore size, and interconnectivity of porous structures. This parameter directly influences

4 transport of soluble particles and indirectly regulates fluid pressure and velocity in tissue

5 engineering scaffolds. The permeability also contributes to the viscoelastic behavior of visco-

6 porous material under loading through frictional drag mechanism. We propose a

7 straightforward experimental method for permeability characterization of tissue engineering

8 scaffolds. In the developed set-up a step-wise spacer was designed to facilitate measurement

9 of the permeability under different compressive strains while maintaining similar experimental conditions during the successive measurements. As illustration of the method,

11 we measured the permeability of scaffolds presenting different average pore sizes and 12 subjected to different compression values. Results showed an exponential relationship 13 between the permeability and the average pore size of the scaffolds. Furthermore, the trend of 14 the permeability decrease with compressive strains was depending on pore sizes of the 15 scaffolds. The permeability also appeared to play a role in relaxation behavior of the scaffolds.

17 Keywords: Scaffolds, Permeability, Pore size, Compressive strain, Frictional drag 


\section{1) Introduction}

Permeability is considered as an integrative variable for tissue engineering (TE) scaffolds reflecting the role of contributing parameters in their architectures such as porosity, pore size, interconnectivity and orientation of pores to flow direction ( $\mathrm{Li}$, de Wijn et al. 2003). The permeability of the scaffold not only influences infiltration and diffusion of soluble particles in TE applications but also passively controls the velocity and pressure fields of fluid content inside the scaffold under mechanical stimulation.

From another point of view, the permeability can be understood as a primary mechanical determinant defining frictional drag that is the resistance of a 3D biomaterial for flowing fluid through its tortuous structure (Mow, Kuei et al. 1980, Nasrollahzadeh and Pioletti 2016). Accordingly, permeability can be controlled to tune viscoelastic behavior of biomaterials under loading. Specifically, energy dissipation and stress relaxation behavior of TE scaffold which are key viscoelastic features in dynamic and static loading regimes, respectively. Interestingly, these mechanobiological variables have been shown to be significantly influential on cellular response of load bearing tissues (Abdel-Sayed, Darwiche et al. 2014, Chaudhuri, Gu et al. 2016).

Obviously, permeability varied under compressive strain due to deformed scaffold internal structure and geometry. Different theoretical models have been developed to establish relationships between the strain and permeability for several tissues such as cartilage (interested reader is referred to (Lai and Mow 1979, Lai, Mow et al. 1981, Holmes 1985, Holmes and Mow 1990)). While many experimental studies have been performed to measure the permeability of biological tissues and scaffolds (see (Pennella, Cerino et al. 2013) for a review), only very few techniques have been developed to quantify the corresponding strain dependent permeability of the scaffolds. In a pioneer work, O’Brien et al (O'Brien, Harley et al. 2007) developed an experimental set-up allowing to measure the permeability of highly 
porous natural scaffolds (porosity higher than 95\%) under different strain conditions. However, their system necessitates disassembly and reassembly steps of the rig for measurements of the permeability under different compressive strains. This technical approach impacts the reliability of the permeability measurement as permeability quantification is highly dependent on the initial and boundary conditions.

Kenneth et al (Ng, Torzilli et al. 2014) tested synthetic PVA scaffolds under confined compression to indirectly quantify strain dependent permeability according to the biphasic theoretical model for cartilage (Lai and Mow 1979). The employed model for permeability determination in that study was based on linear infinitesimal strain theory, while the range of applied compressive strain caused large deformation and nonlinear behavior. Therefore, the predictable permeability values from confined compression experimental data based on the assumed model were not reliable anymore. In particular, Ateshian et al (Ateshian, Warden et al. 1997) has already discussed the limitation of indirect estimation of the permeability from creep or stress-relaxation experiments when finite deformation biphasic theory is used (Ateshian, Warden et al. 1997). They showed low sensitivity of the reaction force vs. time in confined compression (e.g. stress relaxation or creep tests) to large variations of the permeability function parameters. The difficulty of the indirect methods resides in the parameters estimation of the mathematical function for samples with different porosities, pore sizes, interconnectivity and viscoelastic properties, which indeed require several experiments.

In this work, we present a general experimental technique to accurately characterize strain dependent permeability of tissue engineering scaffolds. In the proposed test rig, we designed a step-wise spacer for straightforward characterization of the permeability under different compressions while preserving initial and boundary conditions. As example, we applied this method to recently developed viscoelastic scaffolds presenting different average pore sizes subjected to different compression values. 


\section{2) Materials and Methods}

69 Macroporous scaffolds with different average pore sizes and crosslinking density (3 groups)

70

71

72

73

74

75

76

77

78

79

80

81

82

83

84

85

86

87

$$
\phi=\frac{\left(m_{\text {wet }}-m_{\text {dried }}\right) / \rho_{\text {water }}}{V} \times 100
$$

of pHEMA-EGDMA were prepared by salt leaching method as reported elsewhere (AbdelSayed, Darwiche et al. 2014, Nasrollahzadeh and Pioletti 2016). Each swelled scaffold was cut with a $8 \mathrm{~mm}$ diameter punch and the thickness was sized to $3 \mathrm{~mm}$ using a custom made cutting tool. The equilibrium Young modulus of hydrated scaffolds were determined through applying successive compressive strains (10, 15 and 20\%) with the Instron uniaxial testing machine (Instron E3000, Norwood, Massachusetts, USA) as described elsewhere (Scholten, $\mathrm{Ng}$ et al. 2011). Unconfined stress relaxation tests of $20 \%$ strain were also performed for fine and coarse pore size scaffolds at $50 \mu \mathrm{m} / \mathrm{s}$ or $5 \mu \mathrm{m} / \mathrm{s}$ compression rates as schematically depicted in Fig.1-Left (See supplemental data for detail).

Micro-computed tomography scans (Skyscan 1076, Bruker-microCT, Kontich, Belgium) of freeze-dried scaffolds $(n=3)$ was used to calculate with CTAn software (Bruker-microCT) the average and the distribution of pore sizes in dried state. By measuring the bulk geometry of the scaffolds in hydrated as well as in dried states, the pores volumetric expansion (PVE) was evaluated. We then estimated the corresponding average pore size change as cubic root of PVE ( $\sqrt[3]{P V E}$ ) (Offeddu, Ashworth et al. 2016). Finally, by multiplying this value to obtained results from $\mu \mathrm{CT}$ of dried scaffolds, the hydrated state characteristics were calculated (See supplemental data). The porosity $\phi$ was determined by measuring the volume $V$ and weighting the mass of the swelled $m_{\text {wet }}$ and the dried scaffolds $m_{\text {dried }}$ according to Eq. 1 .
88

We modified a previously proposed set-up by O’Brien et al (O'Brien, Harley et al. 2007) for measuring strain dependent permeability of scaffolds. In the developed set-up as illustrated in 


$$
k=\frac{Q L \mu}{A \rho g h}
$$

106 where, $\mu$ represents the dynamic fluid viscosity (0.001 Pa.s for water), $A$ is the apparent

Fig. 1-Center, we used a step-wise spacer, which allows measurements of the permeability in different compressive strains $(10 \%, 18 \%, 27 \%$, and $35 \%)$ in one assembly.

Before the permeability of scaffold can be measured, we needed to insure that our set-up is leakproof. For this, a thick cylindrical sealant (3.5 mm thickness, $7 \mathrm{~mm}$ internal and $15 \mathrm{~mm}$ outer diameter) was designed to guarantee the sample seal while maintaining a uniform deformation. It was made of a silicon rubber (Elastosoil M4601, Wacker Chemie, Munich, Germany) and we obtained its equilibrium Young modulus by sequential compressive strains of 5, 10, 15 and 20\% considering stress relaxation after each step (Scholten, Ng et al. 2011). The pre-strain resulting in required sealing force to prevent any leakage under the used experimental conditions was evaluated using Hooke’s law and Lamé’s equations for thick walled cylinders.

To calculate the permeability, as schematically shown in Fig.1-Right, after stress equilibrium, the flow rate was measured under constant water head condition using the weight of the flowing water passing the scaffold ( $\mathrm{n}=4-6)$ and a stopwatch. The permeability $k$ of the scaffold was calculated using Eq. 2 based on Darcy’s law (Darcy, Darcy et al. 1856, Pennella, Cerino et al. 2013).

scaffold cross section $\left(\pi r^{2}=2.83 \mathrm{E}-05 \mathrm{~m}^{2}\right), L$ stands for the thickness of the sample $(\mathrm{m}), h$ is the constant fluid head $(2.04 \mathrm{~m}), Q$ denotes the measured volumetric flow rate $\left(\mathrm{m}^{3} / \mathrm{s}\right), \rho$ is the density of the fluid $\left(1000 \mathrm{~kg} / \mathrm{m}^{3}\right.$ for water) and $g$ is the gravity constant $\left(9.81 \mathrm{~m} / \mathrm{s}^{2}\right)$. The thickness of sample decreases in Eq. 2 in case of different compressive strains corresponding to steps of the spacer (3.00, 2.70, 2.45, 2.20 and $1.95 \mathrm{~mm}$, respectively). 


\section{3) Results}

113 We developed different types of viscoelastic scaffold having elastic modulus (0.76 to 1.2

$114 \mathrm{MPa}$ ) within the range of cartilage tissue by tuning the crosslinker $(\mathrm{Cr})$ percentage of the base

115

116

117

118

119

120

121

122

123

124

125

126

127

128

130

131

132

133

134

135

136

material and pore size as reported in Table 1 . The average pore size and porosity for the three types of scaffolds ranged from 162 to $271 \mu \mathrm{m}$ and $63 \%$ to $68 \%$. The obtained results from the stress relaxation tests (Fig. 2- Bottom right) revealed that the initial decay rate and relaxation time of transient stress were significantly different between fine and coarse pore size scaffolds (See supplementary data for detail).

The compressive modulus of the rubber sealant was $1226 \pm 154 \mathrm{kPa}$ and, therefore, with any value higher than $5 \%$ pre-strain of the sealer, leakproof condition for permeability measurement was insured. Typical measurement times varied between 2 to 60 minutes (after reaching steady state condition) depending on the scaffold pore size and applied compression. Performed permeability measurements revealed significant decrease in the permeability as the pore size of the scaffolds was reduced (Fig. 2-top left) and the trend for this reduction is exponential (Fig. 2-top right). Moreover, dramatic permeability drop under compressive strain was observed for scaffolds presenting smaller pore sizes. In fact, for scaffolds with coarse pore sizes $(\mathrm{d}=271 \mu \mathrm{m})$, we observed almost a linear trend for the permeability decrease under compressive strains up to $20 \%$. On the contrary, for scaffolds with the fine pore size (d=162 $\mu \mathrm{m})$, this trend was nonlinear and decreasing exponentially (Fig. 2-bottom left).

\section{4) Discussion}

A fairly simple indeed reliable experimental method for permeability measurement of the scaffolds under compressive strain was presented. Our measurements revealed that the permeability is a exponential function of the average pore size of the scaffolds in strain free condition. Additionally, we observed that the decreasing trend of permeability under compressive strain is quite different depending on pore sizes of the scaffolds. The scaffolds 
137 having fine pore size $(\mathrm{d}=162 \mu \mathrm{m})$ showed an exponential drop in permeability under 138 compression similar to established cartilage model (Lai and Mow 1979, Holmes and Mow 139 1990); coarse pore size scaffolds $(\mathrm{d}=271 \mu \mathrm{m})$, however, indicated almost linear strain 140 dependent permeability reduction. The permeability also appeared to play a role in relaxation

141 behavior of the scaffolds. The release of compression-induced fluid pressure during decaying 142 phase is easier in coarse pore size scaffolds with higher permeability resulting in faster initial 143 decay rate and shorter relaxation time. It is worth mentioning that the complex interaction of 144 entangled chains of polymer network is also reported to be an important mechanism for stress 145 relaxation in covalent crosslinked structures (Mitchell 1980, Kapnistos, Lang et al. 2008).

146 In the present work, we assumed isotropic material properties for our scaffolds since pores are 147 randomly distributed and oriented in the entire the volume (See supplemental data). Our 148 permeability measurements for coarse pore size scaffolds in strain free condition is 149 comparable with reported values by Spain et al (Spain, Agrawal et al. 1998) $\left(1.81 \pm 3.6 \times 10^{-12}\right.$ $150 \mathrm{~m}^{2}$ ) which used synthetic scaffolds with similar characteristics such as porosity range (ranged 151 from 51 to $70 \%$ ) as our scaffolds. On the other hand, the reported values of permeability in 152 O’Brien et al (O'Brien, Harley et al. 2007) study for scaffolds in the range of our fine pore 153 size scaffolds is higher than our measurements. This is not surprising since the porosity of the 154 scaffolds in their study ( $\phi>95 \%)$ is much higher than our scaffolds $(\phi<70 \%)$. It is worth 155 mentioning, according to the semi-empirical Kozeny-Carmen equation (Carman 1956, 156 Pennella, Cerino et al. 2013), the permeability is a function of the porosity of the porous 157 media.

158 We proposed stepwise spacer to eliminate disassembly and reassembly of the rig for 159 measurements of the permeability under different compressive strains. In the proposed set-up 160 of O’Brien et al (O'Brien, Harley et al. 2007), by reinstalling the rig for each measurement, 161 the same experimental condition between different compression values may not be conserved 
162

and, therefore, the obtained results could reflect large variations. In the present set-up, the sealant geometry was designed to have large enough thickness for providing required sealing force after rig assembly. This prevents pressure loss in the chamber. In addition, its small internal radius leads to integration of the sealant and scaffold due to the elastic behavior of the sealant which blocks fluid to pass around the sample. The previously proposed systems (compressing sample edges by sealant axially) for sealing by others such as Sell et al (Sell, Barnes et al. 2008) and O’Brien et al (O'Brien, Harley et al. 2007) is appropriate for soft samples but would damage the structure of stiffer scaffolds under large compressive strain (e.g., 40\%). Likewise, glue solution for sealing is not practical for several reasons including long swelling time after gluing for hydrophobic scaffolds, difficulty for maintaining the amount of glue to prevent water leakage across the edges of the sample and not blocking effective pores of the sample. The main limitation of the present set-up is that with provided $20 \mathrm{kPa}$ pressure head, it is difficult to measure the permeability of scaffolds less than order of $10^{-16} \mathrm{~m}^{2}$ due to very slow flow rate and long required time for passing a detectable amount of water without evaporation. Indeed increasing pressure head is possible, however, in high pressure measurements we should take into the effect of fluid induced strain inside the scaffold (Holmes 1985, Holmes and Mow 1990).

Characterization of strain dependent permeability of tissue engineering scaffolds is critical in load-bearing applications due to its considerable effect on solutes transport, oxygen tension, and pressure and velocity fields as well as scaffolds viscoelastic behavior. All of these biophysical factors may influence cellular differentiation and therefore neo-tissue formation. In our experiments, we identified remarkable differences in permeability trend between scaffolds presenting different averages in pore size. This observation suggests that relying on expressions for permeability as a function of compressive strain such as exponential function 
186 proposed for cartilage may not be good enough predictive for all types of tissue engineering 187 scaffolds. Direct experimental characterization should be preferred.

\section{Acknowledgments}

189 This work was supported by the Swiss National Science Foundation (\#310030_149969 / 1).

190

191 
Abdel-Sayed, P., S. E. Darwiche, U. Kettenberger and D. P. Pioletti (2014). "The role of energy dissipation of polymeric scaffolds in the mechanobiological modulation of chondrogenic expression." Biomaterials 35(6): 1890-1897.

Ateshian, G., W. Warden, J. Kim, R. Grelsamer and V. Mow (1997). "Finite deformation biphasic material properties of bovine articular cartilage from confined compression experiments." Џournal of biomechanics 30(11): 1157-1164.

Carman, P. C. (1956). Flow of gases through porous media, Academic press.

Chaudhuri, O., L. Gu, D. Klumpers, M. Darnell, S. A. Bencherif, J. C. Weaver, N. Huebsch, H.p. Lee, E. Lippens and G. N. Duda (2016). "Hydrogels with tunable stress relaxation regulate stem cell fate and activity." Nature materials 15(3): 326-334.

Darcy, H., H. Darcy and H. Darcy (1856). Les fontaines publiques de la ville de Dijon.

Holmes, M. and V. Mow (1990). "The nonlinear characteristics of soft gels and hydrated connective tissues in ultrafiltration." Journal of biomechanics 23(11): 1145-1156.

Holmes, M. H. (1985). "A theoretical analysis for determining the nonlinear hydraulic permeability of a soft tissue from a permeation experiment." Bulletin of mathematical biology 47(5): 669-683.

Kapnistos, M., M. Lang, D. Vlassopoulos, W. Pyckhout-Hintzen, D. Richter, D. Cho, T. Chang and M. Rubinstein (2008). "Unexpected power-law stress relaxation of entangled ring polymers." Nature materials 7(12): 997-1002.

Lai, W. and V. Mow (1979). "Drag-induced compression of articular cartilage during a permeation experiment." Biorheology 17(1-2): 111-123.

Lai, W., V. C. Mow and V. Roth (1981). "Effects of nonlinear strain-dependent permeability and rate of compression on the stress behavior of articular cartilage." Journal of biomechanical engineering 103(2): 61-66. Li, S., J. R. de Wijn, J. Li, P. Layrolle and K. de Groot (2003). "Macroporous biphasic calcium phosphate scaffold with high permeability/porosity ratio." Tissue Engineering 9(3): 535-548.

Mitchell, J. (1980). "The rheology of gels." Journal of Texture Studies 11(4): 315-337.

Mow, V. C., S. Kuei, W. M. Lai and C. G. Armstrong (1980). "Biphasic creep and stress relaxation of articular cartilage in compression: theory and experiments." Journal of biomechanical engineering 102(1): 73-84.

Nasrollahzadeh, N. and D. P. Pioletti (2016). Development of dissipative scaffolds with different mechanisms of dissipation. In the procceeding of 10th International Conference on the Mechanics of Time Dependent Materials,. Paris-France.

Ng, K. W., P. A. Torzilli, R. F. Warren and S. A. Maher (2014). "Characterization of a macroporous polyvinyl alcohol scaffold for the repair of focal articular cartilage defects." Journal of tissue engineering and regenerative medicine 8(2): 164-168.

O'Brien, F. J., B. A. Harley, M. A. Waller, I. V. Yannas, L. J. Gibson and P. J. Prendergast (2007). "The effect of pore size on permeability and cell attachment in collagen scaffolds for tissue engineering." Technology and Health Care 15(1): 3-17.

Offeddu, G., J. Ashworth, R. Cameron and M. Oyen (2016). "Structural determinants of hydration, mechanics and fluid flow in freeze-dried collagen scaffolds." $\underline{\text { Acta }}$ biomaterialia.

Pennella, F., G. Cerino, D. Massai, D. Gallo, G. F. D. U. Labate, A. Schiavi, M. A. Deriu, A. Audenino and U. Morbiducci (2013). "A survey of methods for the evaluation of tissue engineering scaffold permeability." Annals of biomedical engineering 41(10): 20272041. 
241 Scholten, P. M., K. W. Ng, K. Joh, L. P. Serino, R. F. Warren, P. A. Torzilli and S. A. Maher 242 (2011). "A semi - degradable composite scaffold for articular cartilage defects." Journal 243 of Biomedical Materials Research Part A 97(1): 8-15.

244 Sell, S., C. Barnes, D. Simpson and G. Bowlin (2008). "Scaffold permeability as a means to 245 determine fiber diameter and pore size of electrospun fibrinogen." Journal of Biomedical 246 Materials Research Part A 85(1): 115-126.

247 Spain, T., C. Agrawal and K. Athanasiou (1998). "New technique to extend the useful life 248 of a biodegradable cartilage implant." Tissue engineering 4(4): 343-352. 


\section{Tables}

\begin{tabular}{c|ccc} 
Scaffold type & $\begin{array}{c}\text { 4\%Cr-Fine } \\
\text { pore size }\end{array}$ & $\begin{array}{c}\text { 6\%Cr-Medium } \\
\text { pore size }\end{array}$ & $\begin{array}{c}\text { 8\%Cr0Coarse } \\
\text { pore size }\end{array}$ \\
\hline Equilibrium elastic modulus & $760 \pm 63$ & $1008 \pm 163$ & $1201 \pm 154$ \\
Time to relax 90\% of transient stress at $\mathbf{5} \boldsymbol{\mu \mathrm { m }} / \mathbf{s}$ rate & $581.5 \pm 76$ & $378 \pm 30$ & $341 \pm 45.5$ \\
Average pore size $-\boldsymbol{d}(\boldsymbol{\mu m})$ & $162 \pm 5$ & $196 \pm 4$ & $271 \pm 5$ \\
Range of distribution of pore sizes within $\pm \boldsymbol{\sigma}(\boldsymbol{\mu m})$ & $107-216$ & $127-266$ & $175-367$ \\
Porosity $-\phi(\%)$ & $68 \pm 2$ & $66 \pm 3$ & $63 \pm 3$
\end{tabular}

Table 1. Important characteristics of the developed viscoelastic scaffolds. 


\section{Figures captions}

Figure 1. Left: Compression test of the sealant for calculating required compressive strain to prevent leakage. Centre: Permeability chamber with stepwise spacer and the sealant. Right: Schematics of the experimental setup to measure permeability of a scaffold under different compressive strains.

Figure 2. Results of permeability and rate dependent stress relaxation measurements for viscoelastic scaffolds under compressive strain. Top-Left: Comparison of permeability for different groups of scaffolds; plus $(+)$ symbol shows significant difference $(\mathrm{p}<0.05)$ using Student t-test ( $n=4-6)$. Top- Right: Trend of permeability with average pore size of the scaffolds in $0 \%$ strain condition. Bottom- Left: Strain dependent permeability trend for fine and coarse pore size scaffolds up to $20 \%$ strain. Bottom-Right: Representative stress relaxation curves of $20 \%$ strain for fine and coarse pore size scaffolds at 5 and $50 \mu \mathrm{m} / \mathrm{s}$ loading rates. 

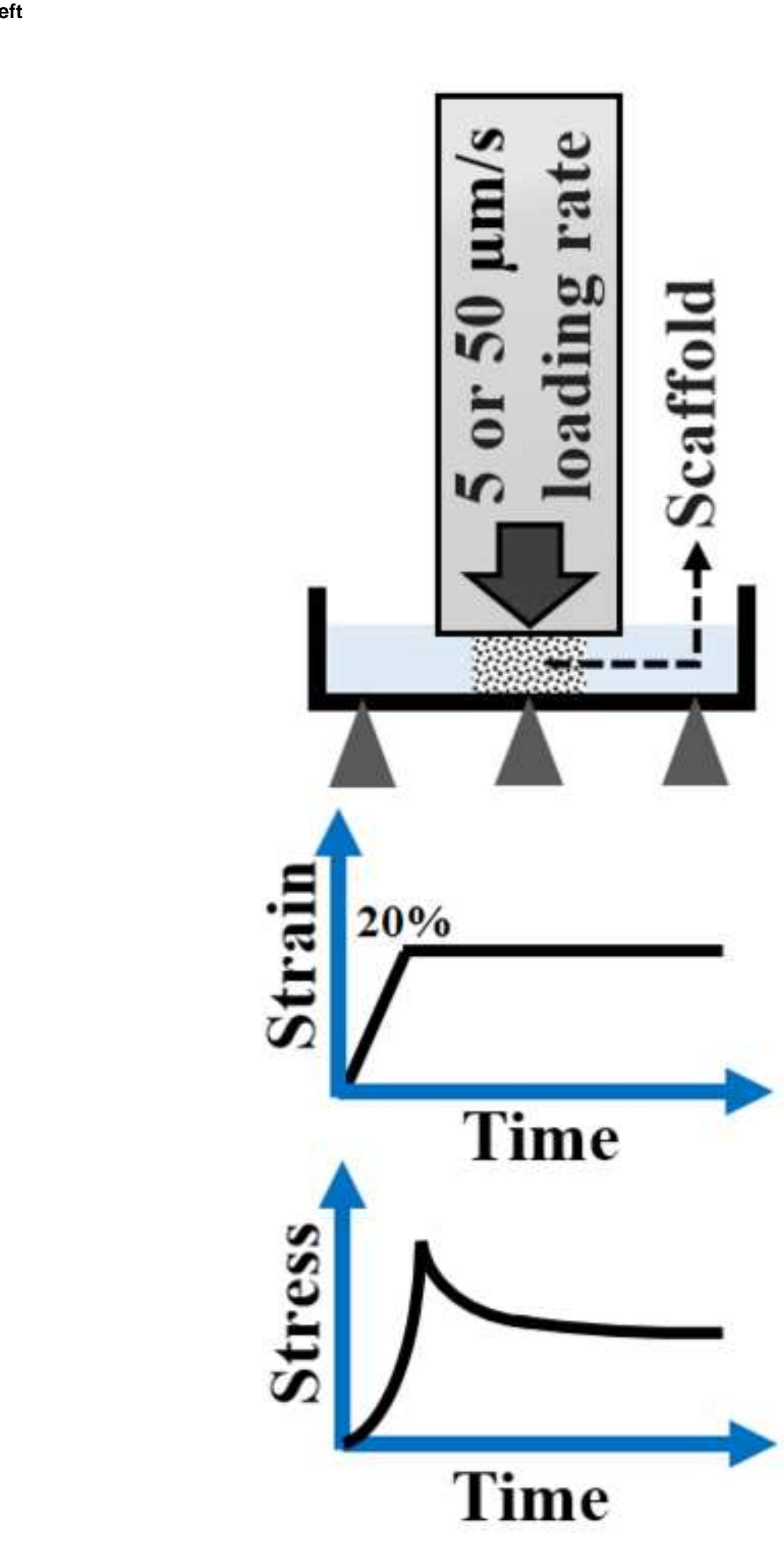

Figure 1-left

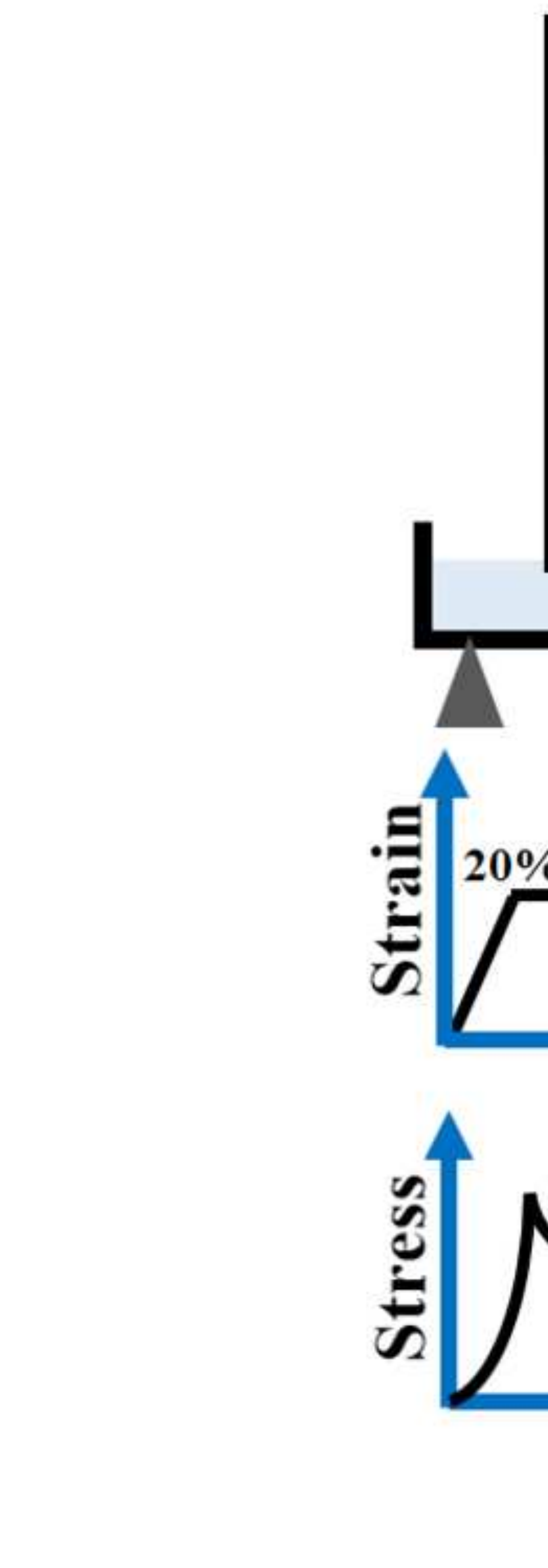

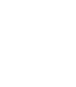

t.
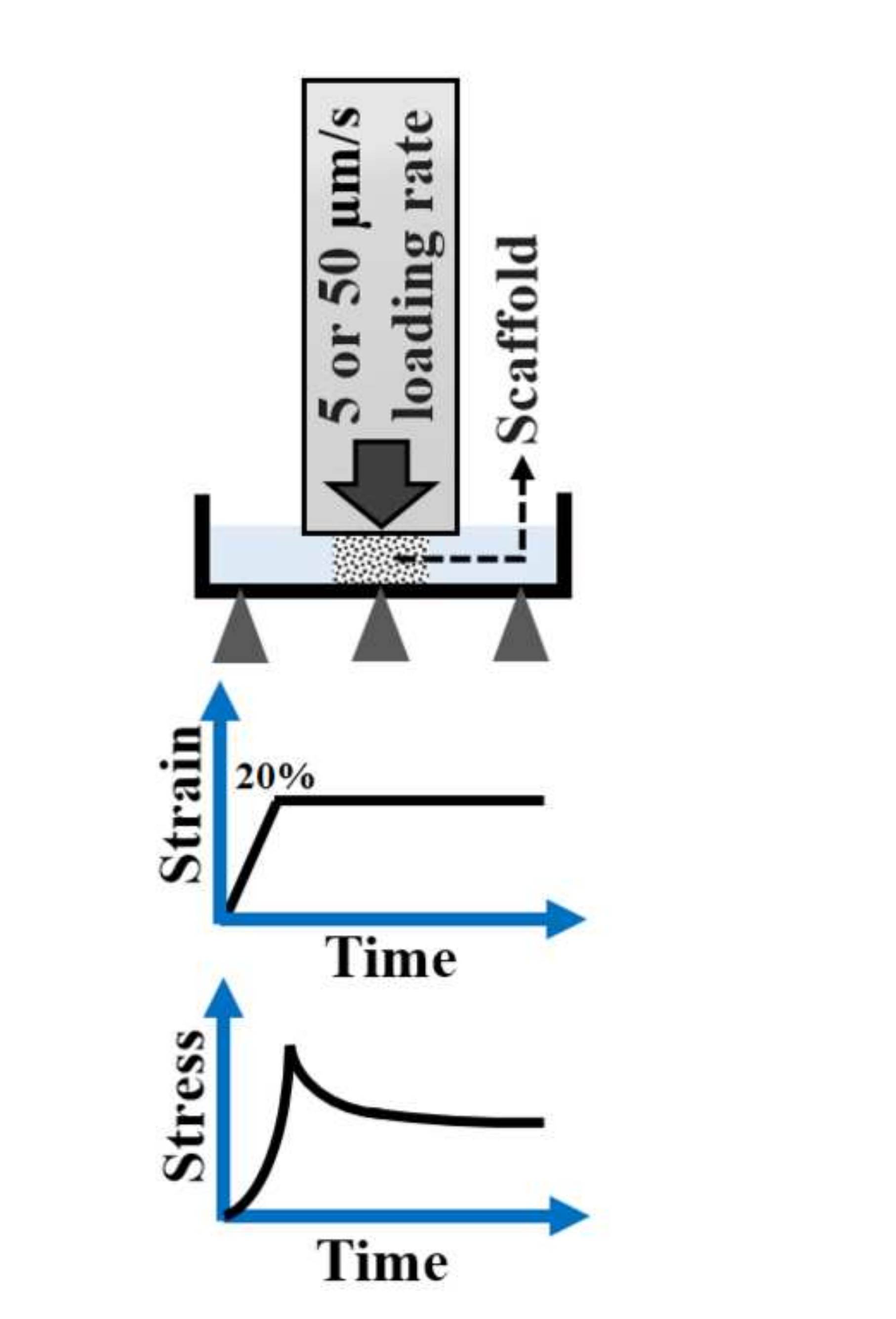

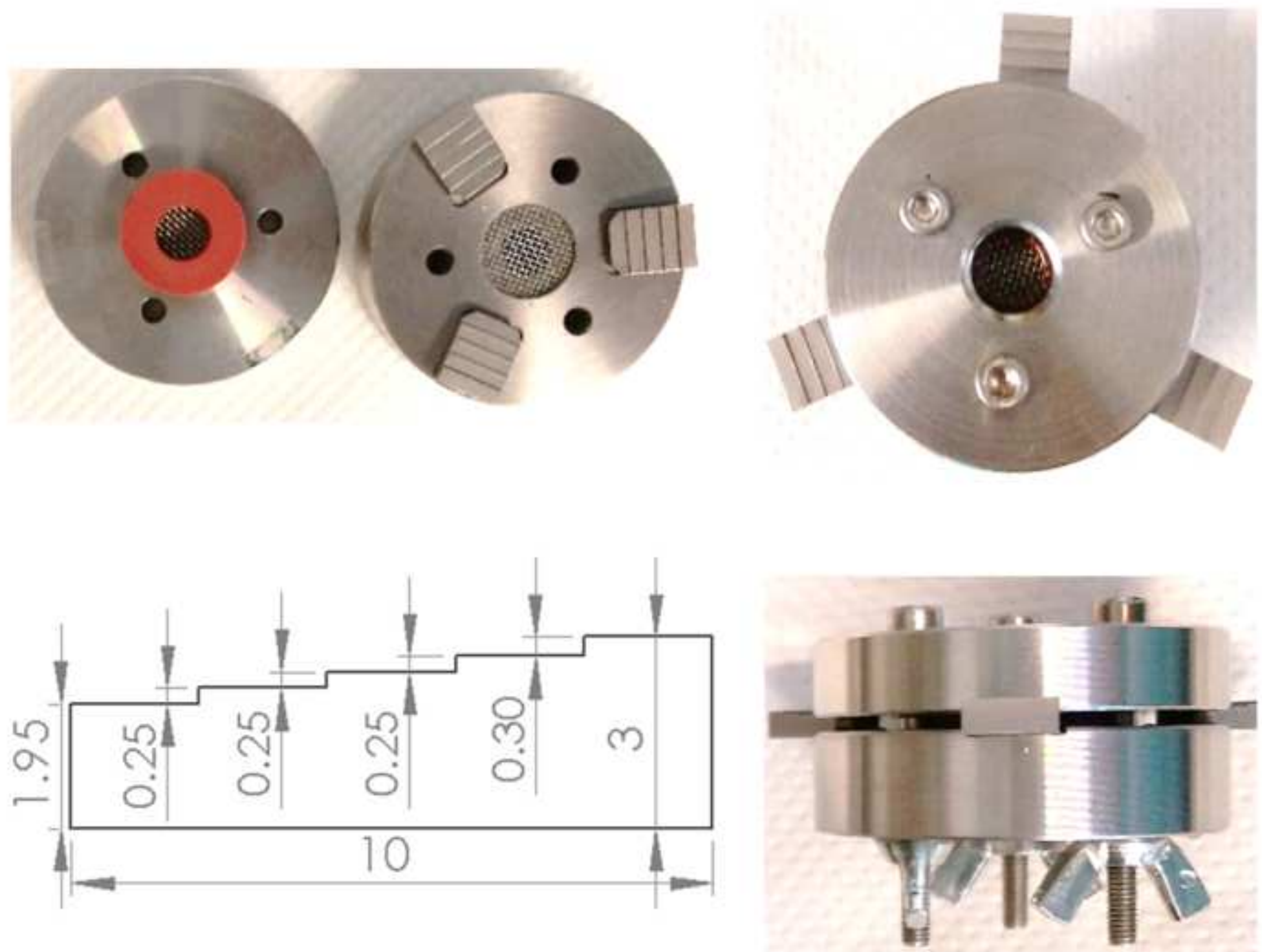


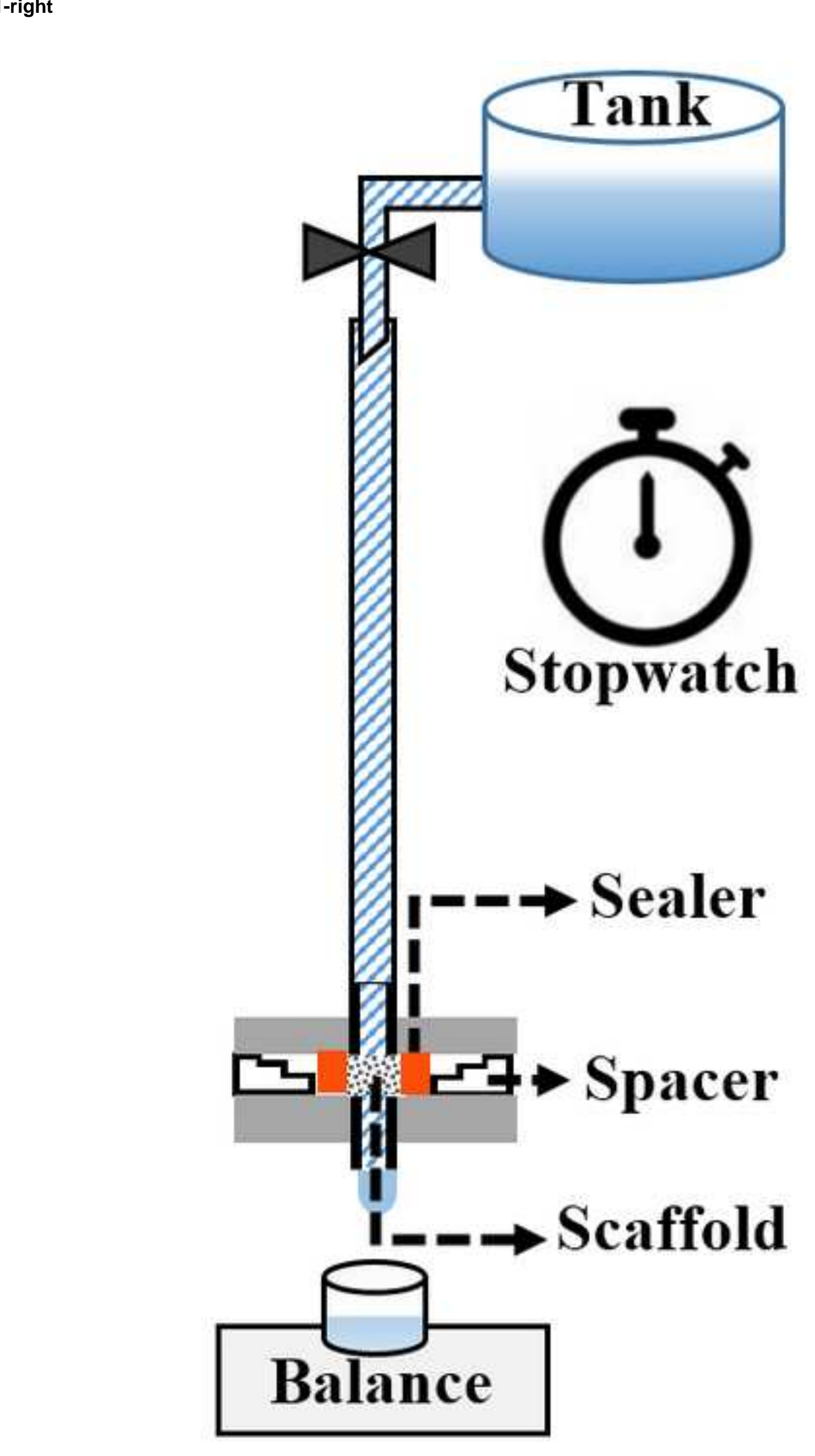

Figure 1-right
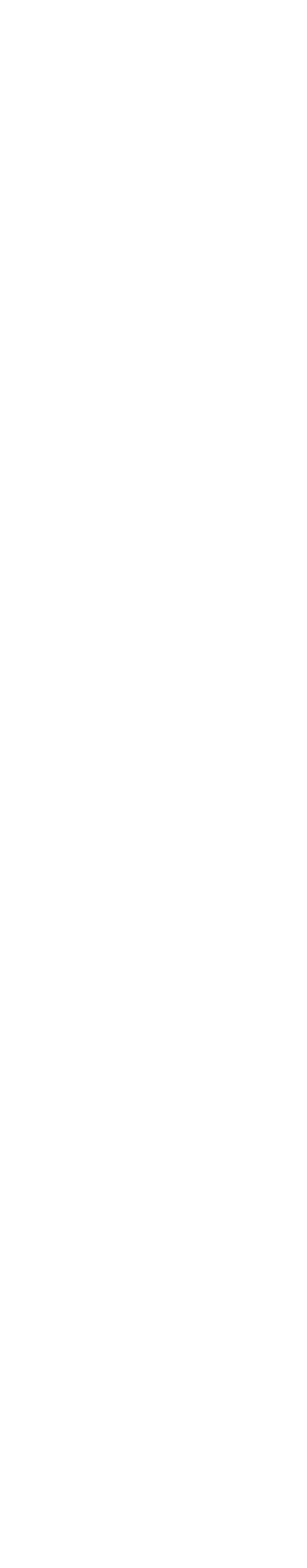


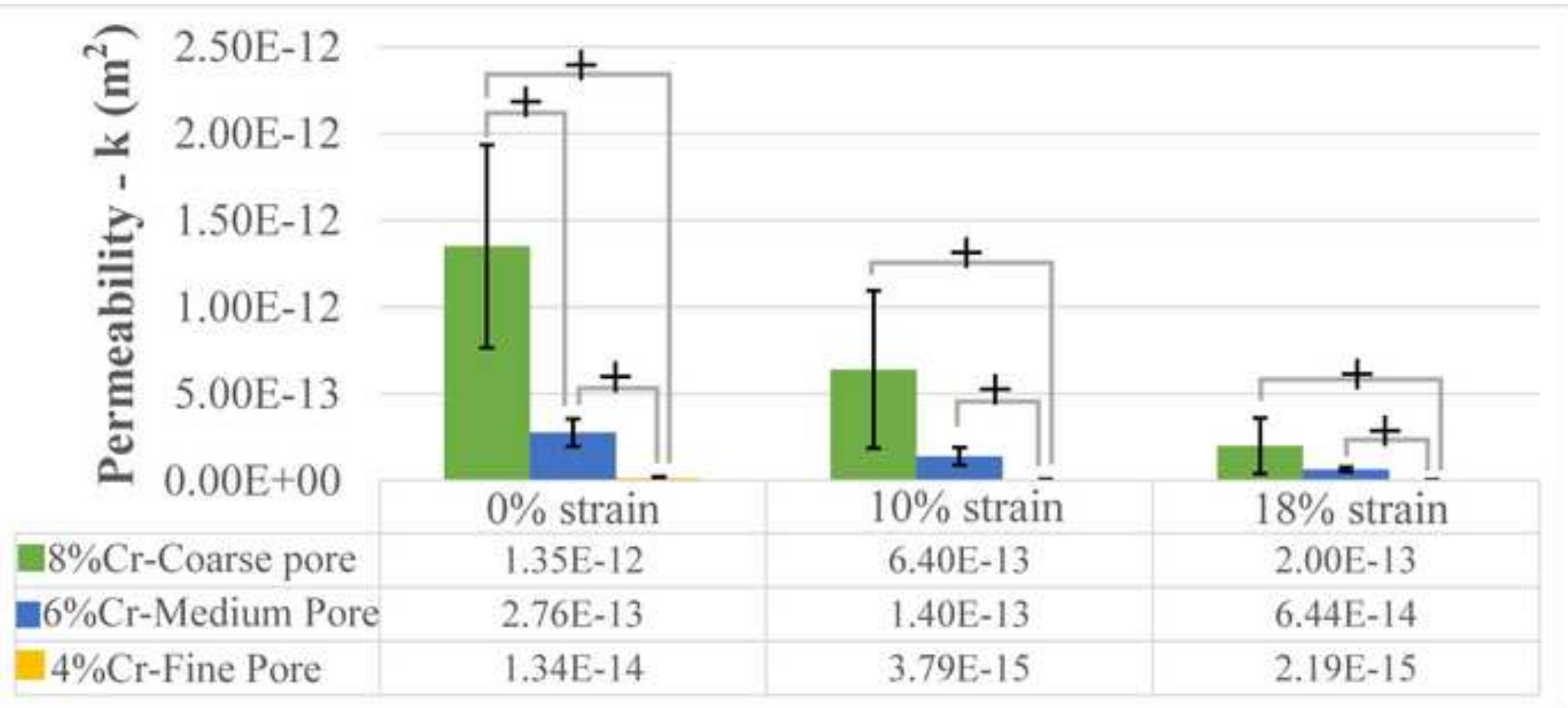




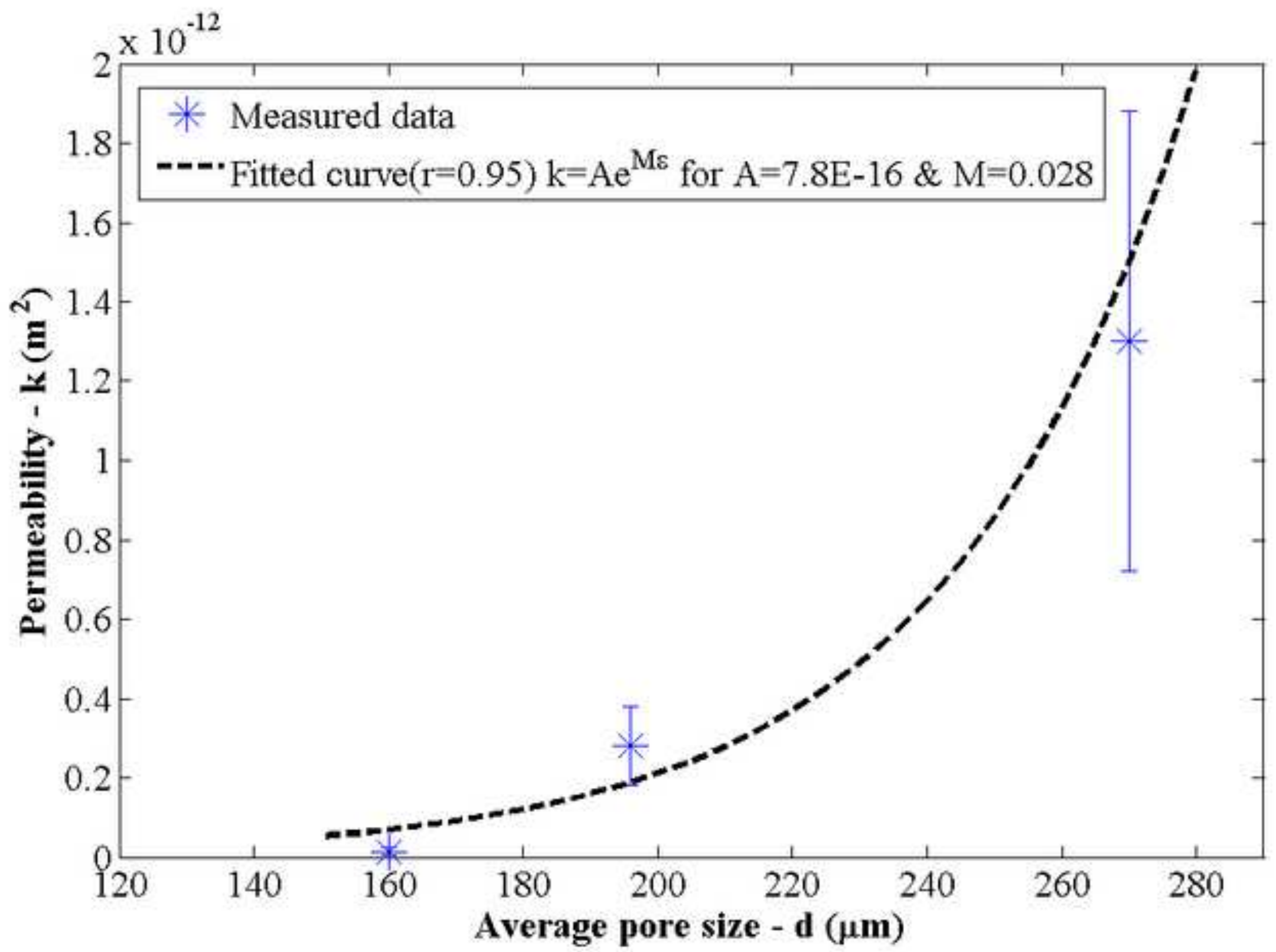



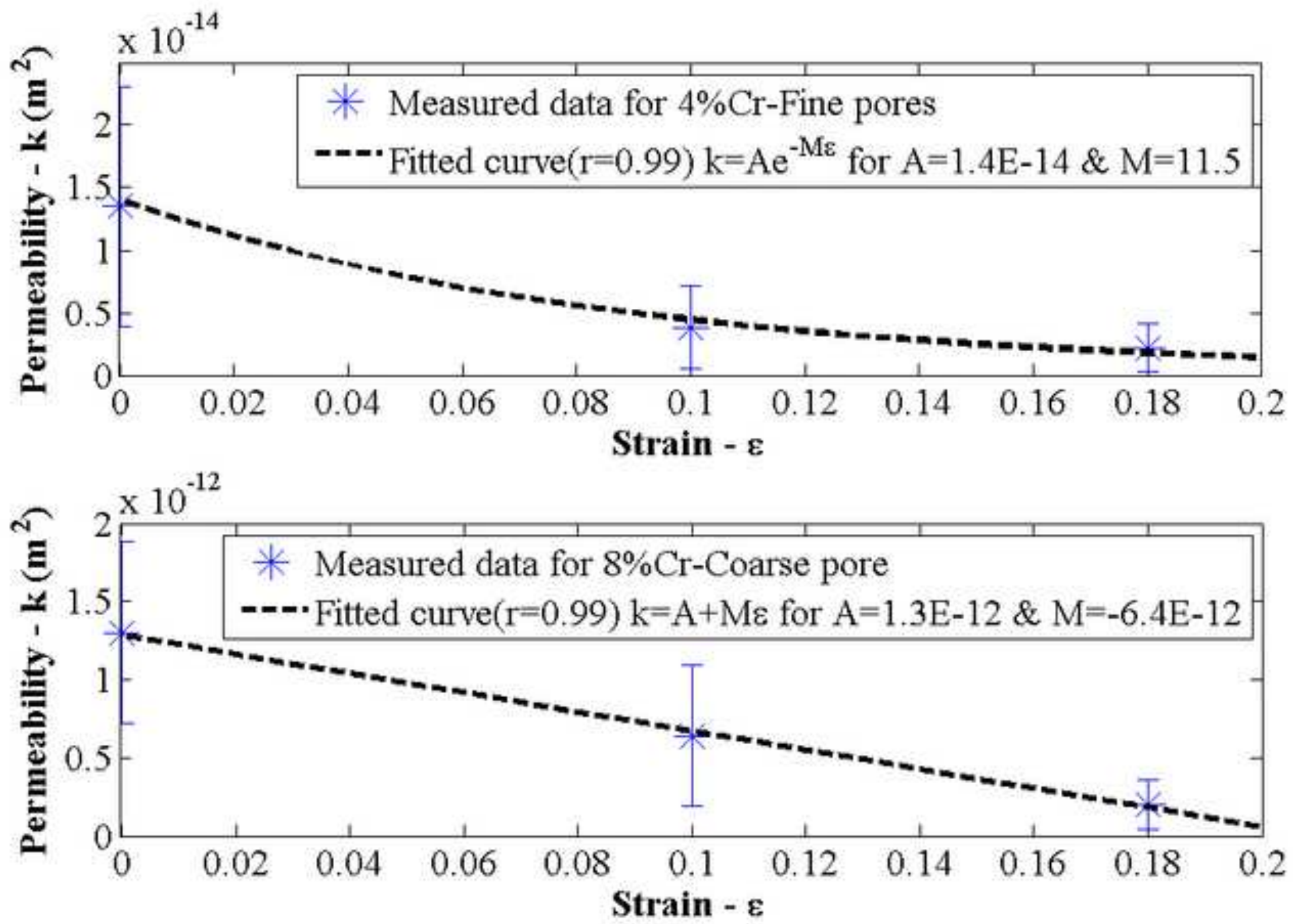


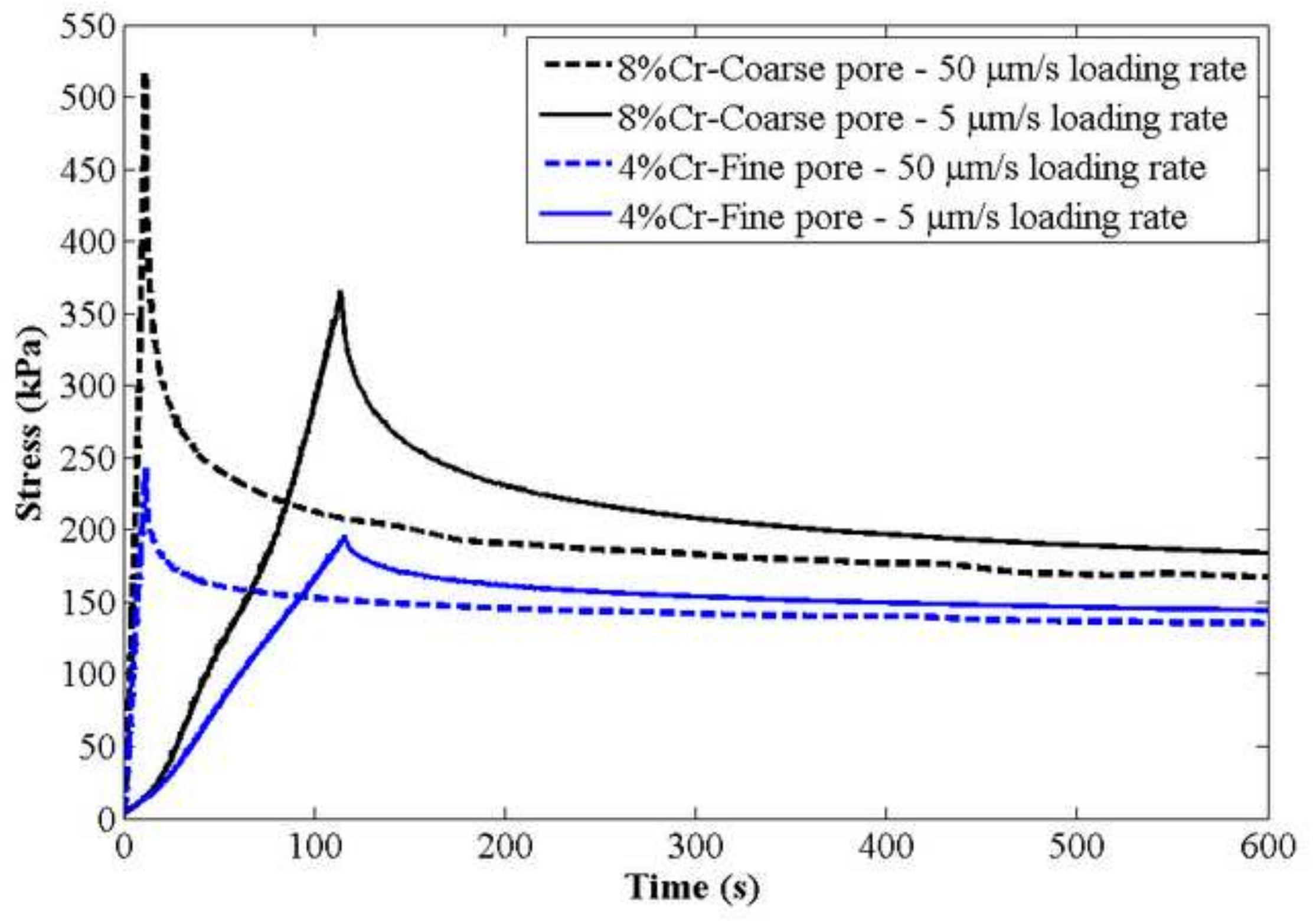

